\title{
PERSISTENCE OF ANTIBIOTICS IN BLOOD OF PATIENTS WITH ACUTE RENAL FAILURE. III. PENICILLIN, STREPTOMYCIN, ERYTHROMYCIN AND KANAMYCIN * $\dagger$
}

\author{
By CALVIN M. KUNIN $\ddagger$ AND MAXWELL FINLAND \\ (From the Thorndike Memorial Laboratory, Second and Fourth (Harvard) Medical Services, \\ Boston City Hospital, and the Department of Medicine, Harvard Medical \\ School, Boston, Mass.)
}

(Submitted for publication April 7, 1959; accepted May 1, 1959)

In the preceding papers, one dealing with tetracycline and chlortetracycline (2) and the other with chloramphenicol (3), the circumstances under which these drugs would be expected to accumulate in the blood of patients with renal failure were indicated. Dosage regimens were outlined which took into account the limitations imposed by renal disease on the elimination of these drugs. The present report presents the results of studies on the persistence of four additional antibiotics; penicillin, streptomycin, erythromycin and kanamycin, following their administration to patients with severe renal failure. Since anuria may be considered to represent the maximum impairment of renal function as seen in man, emphasis was placed on the study of patients in this state in determining the maximum contribution of nonrenal factors toward the elimination or inactivation of these drugs under the physiological limitations of uremia. The findings should prove helpful in delineating the optimum dosage regimens for these drugs in patients with markedly impaired renal function.

\section{MATERIALS AND METHODS}

Definitions. Anuria is defined, for the purpose of this study, as the condition in which uremic patients excreted less than $400 \mathrm{ml}$. of urine per day for three or more days; patients who excreted somewhat more than $400 \mathrm{ml}$. per day are referred to as oliguric and when the urinary volume was in excess of $1,500 \mathrm{ml}$. per day they were considered to be in the diuretic phase.

The serum half-life $(T / 2)$ of each antibiotic was determined by the method of least squares (4).

* Supported in part by Research Grant E-23 from the National Institutes of Health, Bethesda, Md.

$\dagger$ A summary of some of these findings has appeared (1).

$\ddagger$ Postdoctoral Fellow, National Institute of Allergy and Infectious Diseases. Present address: Department of Preventive Medicine, University of Virginia, Charlottesville, Va.
Patients studied 1 and antibiotics used. Penicillin was given to 13 patients, including eight with severe renal failure and five without significant renal impairment; two of the anuric patients also had severe liver disease secondary to nutritional cirrhosis. The antibiotic was administered as a single injection of an aqueous solution of penicillin $G$ and given as the potassium salt by the intramuscular route to three patients and intravenously as the sodium salt (dissolved in $20 \mathrm{ml}$. of distilled water or physiological saline solution and injected over a period of five to 10 minutes) to nine others. The remaining patient was studied after a course of procaine penicillin G. Streptomycin was given as a single intramuscular injection of $1.0 \mathrm{Gm}$., as the sulfate, to four severely uremic patients and to four control patients who had normal renal function. One uremic patient received $1.5 \mathrm{Gm}$. intramuscularly in a single injection and another uremic patient was given $3.5 \mathrm{Gm}$. over a three day period. Erythromycin was given as the glucoheptonate 2 to three uremic patients and to two controls; $0.5 \mathrm{Gm}$. of erythromycin base equivalent was dissolved in $10 \mathrm{ml}$. of distilled water and added to $100 \mathrm{ml}$. of 5 per cent dextrose in water solution and injected intravenously over a period of 15 to 20 minutes. Kanamycin ${ }^{3}$ was given by the intramuscular route to two severely uremic patients and two controls in a dose of $1 \mathrm{Gm}$. as the sulfate.

Assay of antibiotics in serum. ${ }^{4}$ Venous blood was drawn prior to the administration of each antibiotic and also one to two hours after completion of the injection and at convenient intervals thereafter. The blood was permitted to clot and the serum was separated and stored at $-20^{\circ} \mathrm{C}$. until the time of the assay. Levels of penicillin in serum were determined by the twofold dilution method in brain heart infusion broth (Difco, $\mathrm{pH}$ approximately 7.4) using Streptococcus 98 as the test organism, $0.5 \mathrm{ml}$. of a $10^{-4}$ dilution of the fully grown culture being added

$1 \mathrm{We}$ are indebted to Dr. John P. Merrill for permission to study some of the patients on the Cardiorenal Service of the Peter Bent Brigham Hospital, and to Dr. Searle B. Rees for his cooperation in obtaining some of the specimens of blood from those patients.

2 Kindly supplied by Eli Lilly and Company as Ilotycin Glucoheptonate ${ }^{\circledR}$.

3 Supplied by the Bristol Laboratories as Kantrex ${ }^{\circledR}$.

${ }^{4}$ Clare Wilcox and Ann Najarian performed some of these assays. 
to an equal volume of serum dilutions. Streptomycin was assayed in serum by the cup-plate method (5) using $B$. subtilis as the test organism. In the case of Patient $\mathrm{Su}$, who had also received penicillin, sera were assayed by the twofold dilution method using as the test organism a strain of Klebsiella pneumoniae that was sensitive to 2 $\mu \mathrm{g}$. per $\mathrm{ml}$. of streptomycin and resistant to $64 \mu \mathrm{g}$. per $\mathrm{ml}$. of penicillin. Comparable results were obtained when some of these sera were also assayed by the $B$. subtilis cup-plate method in the presence of 100 units per ml. of penicillinase. ${ }^{5}$ Levels of erythromycin in serum were determined by both the twofold dilution method in broth and the agar-cup-plate method (5), employing Sarcina lutea as the test organism in each instance. Assay of erythromycin in the sera of one patient who had received a previous course of tetracycline therapy was performed by the twofold dilution method employing a freshly isolated strain of Staphylococcus aureus that was resistant to $62.5 \mu \mathrm{g}$. per $\mathrm{ml}$. of tetracycline and sensitive to 0.12 $\mu$ g. per $\mathrm{ml}$. of erythromycin.

Assays of serum for kanamycin were done by the cupplate method using $B$. subtilis (ATCC 6633) obtained from Bristol Laboratories and a modified streptomycin technique (5). Duplicate assays for kanamycin were done in this laboratory and by the Bristol Laboratories ${ }^{6}$ and the results were in close agreement.

RESULTS

\section{Penicillin}

The data obtained in eight patients with severe renal disease and in five others who had normal renal function are presented in Table I; five of the former were anuric (as defined). The mean pencillin half-life in five patients with normal renal function who received the dose of $1,000,000$ units of penicillin intravenously was $0.56 \pm 0.17$ hour; these values are similar to those observed by others (6). The serum decay rates expressed as per cent fall per hour \pm 1 standard error are also presented in the table in order to demonstrate the inherent variability of the data.

The half-life of penicillin in the serum in $\mathrm{Gi}$ and $\operatorname{Re}$ during the anuric phase was 10.1 and seven and two-tenths hours, respectively. $\mathrm{Pa}$ tient Vo, who was totally anuric and in the terminal phase of hepatic cirrhosis, had marked ascites and jaundice; her serum bilirubin levels ranged from 18.0 to $20.7 \mathrm{mg}$. per cent during the study period. The penicillin half-life in this patient was 30.5 hours, the most prolonged ob-

\footnotetext{
5 Supplied by Schenlabs Pharmaceutical, Inc., New York, N. Y., as Neutrapen®.

${ }^{6}$ Courtesy of Dr. Kenneth Hubel.
}

served in this group, even longer than that of $\mathrm{Pa}$ tient $\mathrm{Fi}$ (21.2 hours) who had received procaine penicillin and was totally anuric. Patient Do also had severe liver failure and was in the anuric category but was excreting about $100 \mathrm{ml}$. of urine per day. The serum half-life of penicillin in this patient was 16.4 hours which is significantly longer than in the anuric patients without severe liver disease after a dose of aqueous penicillin G.

Three patients ( $\mathrm{Gi}, \mathrm{Si}$ and $\mathrm{Ma}$ ) were studied during the oliguric or early diuretic phases of their disease. At these times, the half-life of penicillin in the the serum of these patients was less than in the anurics, ranging from four to 10.6 hours. Three patients ( $\mathrm{Gi}, \mathrm{Re}$ and $\mathrm{Su}$ ) were studied during the diuretic phase when the penicillin half-life ranged from three and ninetenths to six and one-half hours. One individual who was followed through to early recovery (Gi) still demonstrated an abnormal disappearance curve with a half-life of six and three-tenths hours one month after onset of the anuria. Patient $\mathrm{Su}$, who subsequently died of acute tubular necrosis and severe uremia, had a mean penicillin half-life of only three and one-half hours at the time of the last observations one month after the onset of anuria and shortly before death.

It can be seen from these data that Patients Vo and Do, who had severe hepatic impairment as well as anuria, differ markedly from the other patients in their inability to clear aqueous penicillin from their serum. They had the longest half-life and slowest rate of decay of penicillin in their serum. ${ }^{7}$

\section{Streptomycin}

Patient $\mathrm{Su}$, who was studied while receiving penicillin, also was given $3.5 \mathrm{Gm}$. of streptomycin intramuscularly over a three day period shortly after completion of the first penicillin study. During the subsequent 20 days after the last dose of streptomycin, a number of specimens of serum were obtained and stored and all of them were subsequently assayed together for both penicillin and streptomycin. The results are presented in Figure 1. During these 20 days, the patient was

7 The only similarly prolonged half-life was in Patient $\mathrm{Fi}$, but this patient received procaine penicillin $\mathrm{G}$ intramuscularly and the prolonged half-life is, in part, the result of the repository effect. 


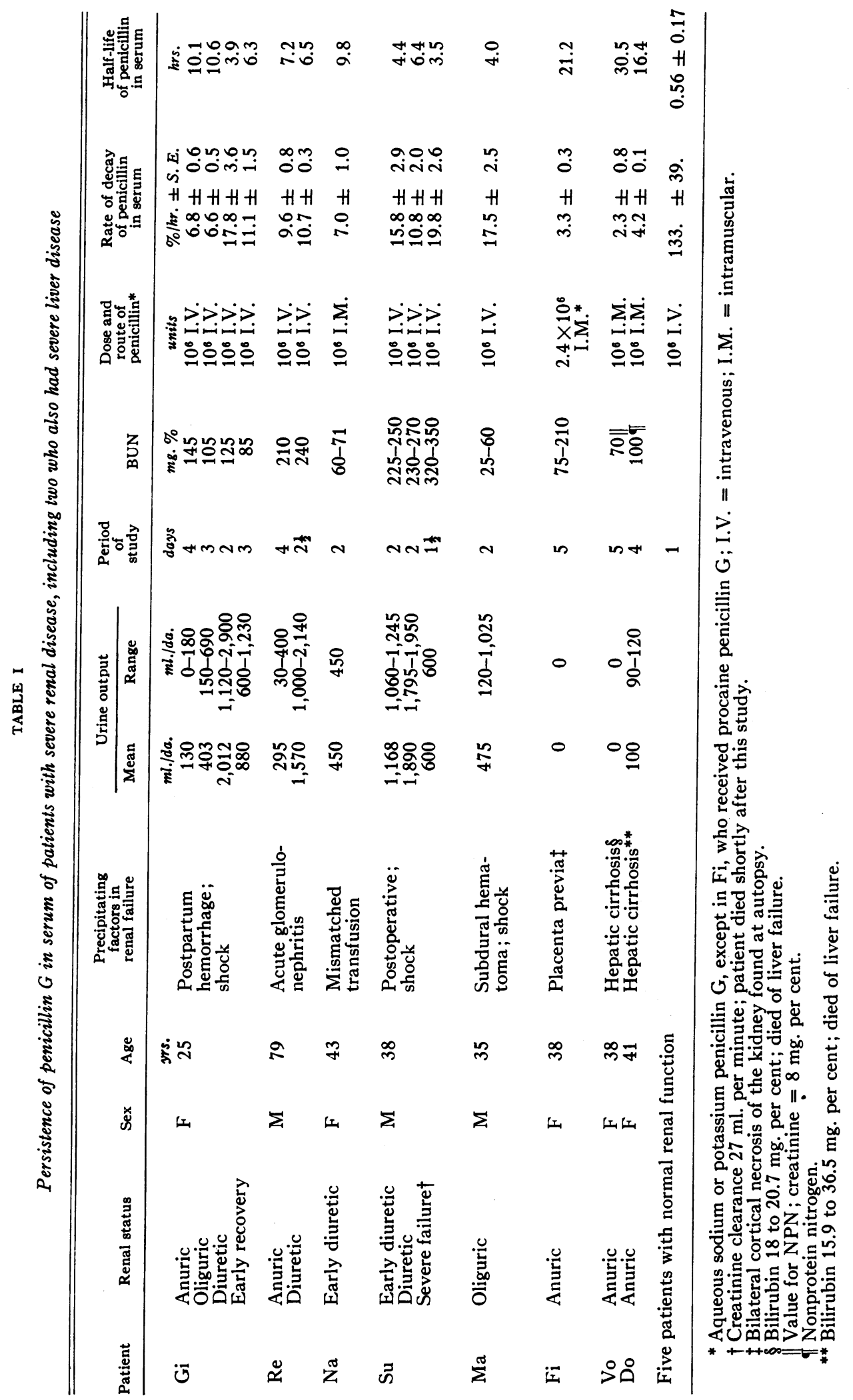




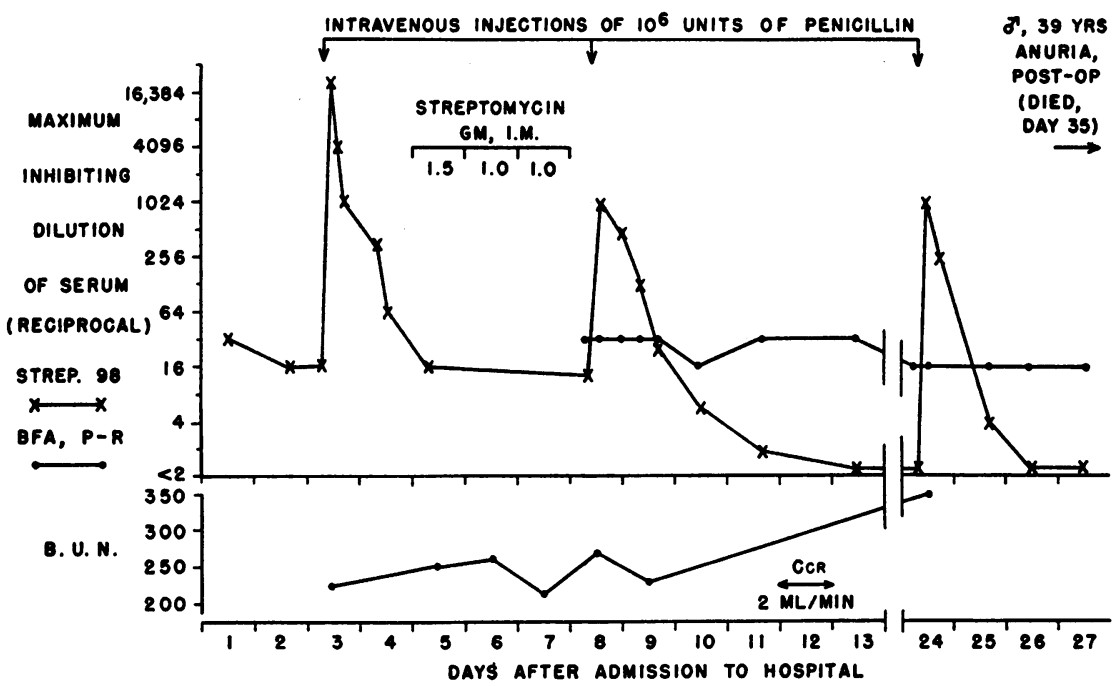

Fig. 1. Penicillin and Streptomycin Activity in Serum of an Anuric Patient After Injections of These Antibiotics

Activity of penicillin was determined by assay with Streptococcus 98 that was moderately resistant to streptomycin. Activity of streptomycin was assayed with a penicillin resistant strain of Klebsiella pneumoniae (BFA-PR).

not anuric but his creatinine clearance was only $2 \mathrm{ml}$. per minute. The very slow fall in levels of streptomycin in the serum is quite striking particularly when compared to the decay rate of penicillin. The data presented in this figure are based on twofold dilution assays, but assays repeated with the cup-plate method using $B$. subtilis and penicillinase gave similar results.

Data in five other severely uremic patients and in four normal subjects all of whom received streptomycin are presented in Table II. It is evident that this drug was retained for long periods in the uremic patients The longest halflife observed was in Patient Go when she was anuric. The variations in the half-life noted in the three patients who were studied during the diuretic phase reflect the rate of recovery in these individuals.

\section{Erythromycin}

Table III shows data obtained in three patients with acute renal failure and in two patients with normal renal function, all of whom were given an intravenous dose of $500 \mathrm{mg}$. of erythromycin as the glucoheptonate. The halflife of erythromycin in the serum was one and two-tenths and one and six-tenths hours in the two control patients and ranged from four to five and eight-tenths hours in the anuric patients, including the one who was studied in the diuretic stage. These data suggest that nonrenal factors also play an important role in the clearance of this drug. Of interest is the finding that the halflife of erythromycin in the serum in $\mathrm{Re}$, the patient with acute toxic hepatitis due to carbon tetrachloride, was not more prolonged than that observed in patients with anuria in whom liver function was apparently not impaired. The serum bilirubin level in this patient was $4.1 \mathrm{mg}$. per cent shortly before the erythromycin injection.

\section{Kanamycin}

Kanamycin levels in the serum were determined after a single intramuscular injection of $1 \mathrm{Gm}$. in two severely uremic patients and in two patients without renal functional impairment. The results are presented in Figure 2. The serum half-life in the controls was about four hours and it was greatly prolonged in the uremic patients. The half-life of kanamycin in the uremic patients was not calculated because of the wide fluctuations in the serum levels possibly due to shifts of water from various body compartments (both patients were edematous and receiving intravenous fluids) and possibly also because of irregular absorption of the drug from the intramuscular in- 


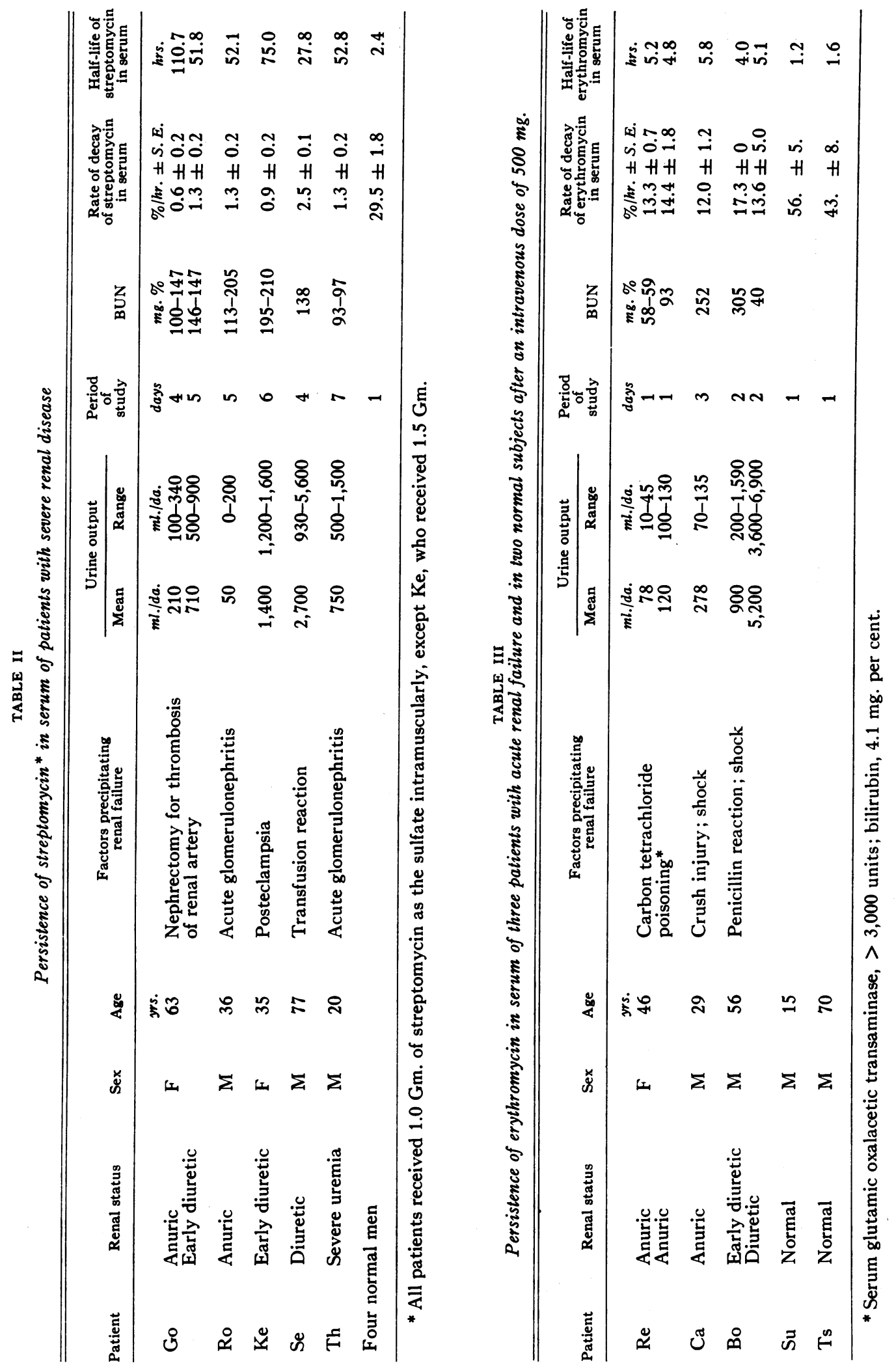




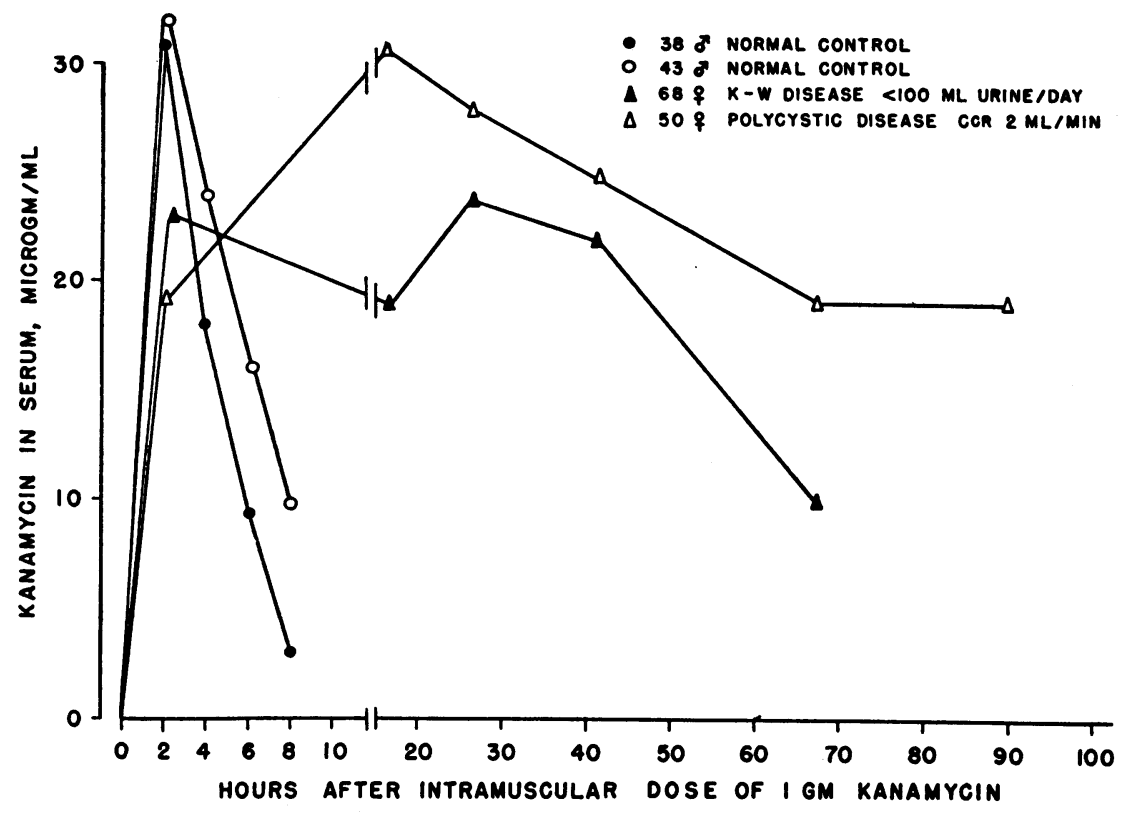

Fig. 2. Levels of Kanamycin in Serum After Intramuscular Injection of 1.0 Gm. in Two Uremic Patients and in Two Subjects with Normal Renal FUNCTION

jection. It will be noted from this figure that rather high levels were still present in the serum three days after the injection of a single dose.

\section{DISCUSSION}

\section{Penicillin}

The clearance of penicillin in normal individuals is very rapid and is a combined function of glomerular filtration and active tubular transport (7). The renal removal of the drug is so efficient that other clearance mechanisms cannot ordinarily be considered of any consequence. In 1944, Humphrey (8) reported observations on two anuric women who had been given a single injection of aqueous penicillin G. He was unable to recover the drug in the small amount of urine excreted, but demonstrated penicillin activity in the serum for up to five days in each of these patients. Barnett, McNamara, Shultz and Tompsett (9) attributed the slower clearance of penicillin in premature infants to their limited renal function. In their studies, the ratio of the clearance of penicillin to that of inulin was 2.1 in prematures as compared with 4.4 in older children. Studies by Huang and High (10) in children of various ages given penicillin also demonstrated that premature and normal full term infants cleared penicillin much less rapidly than older infants and children. The renal clearance of penicillin by the aged is also decreased, paralleling decreases in renal function with age (11-13).

Beyer, Boger and co-workers (14) demonstrated that caronamide reduced the renal clearance of penicillin to approximately the glomerular filtration rate in normal subjects. Their data did not appear to indicate the presence of mechanisms of clearance of the penicillin other than renal excretion. However, in the experiments of Rowlands, Rowley and Stewart (15) who used radioactive sulfurlabeled penicillin in cats, only about 36 to 66 per cent of the administered dose was recovered in the urine as the active drug; the remainder consisted of unknown degradation products.

Studies by Eagle (16) on the in vitro inactivation of penicillins indicated that penicillin $G$ is inactivated by human serum at a rate of 10 per cent per hour when incubated at $37^{\circ} \mathrm{C}$. This corresponds to a half-life of about seven hours and is considerably more rapid than the value of 60 hours reported for inactivation in aqueous solutions of penicillin at $\mathrm{pH} 7.5$ at $37^{\circ} \mathrm{C}$. (17).

Anderson and Broderson (18) studied the velocity constants of the clearance of penicillin 
from the plasma of dogs; following bilateral nephrectomy, the mean velocity constant fell from a normal value of 1.70 to 0.41 "reciprocal hours." The rate then fell to zero in animals which had been completely eviscerated or had combined nephrectomy and hepatectomy. These results would indicate that the liver rather than the plasma accounts for the major nonrenal clearance of the drug in the dog. The same would appear to be true in man, since in Patients Vo and Do, who had severe disease of both the liver and the kidneys, the half-life of aqueous penicillin $\mathrm{G}$ was the most prolonged, namely 30.5 and 16.4 hours, respectively. The half-life in the two anuric patients without hepatic disease was seven and two-tenths and 10.1 hours, which is somewhat greater than that found by Eagle in his in vitro studies with human serum (16). Since both of these patients were still excreting some urine, these values must be considered to be even less than would be expected in a totally anuric subject.

Thus it would appear that there is only a fair correlation between the in vitro serum studies and the in vivo findings. A possible explanation for these seemingly divergent data may lie in the fact that only a part of the total body penicillin store is in contact with plasma at any time. Spitzy and Hitzenberger (19) reported that the relative distribution volume of penicillin $G$ is $0.56 \pm 0.17$ $\mathrm{ml}$. per $\mathrm{Gm}$. body weight in humans, indicating that the drug must be distributed in a relative volume that is about 10 times greater than the intravascular compartment. Also, protective substances may be present in the body which are not detected in the in vitro experiments.

From the therapeutic point of view Bull (20) indicated that a single injection of penicillin would suffice for prolonged periods in the anuric patient. The data presented in the present study would indicate that, in the anuric patient, a single injection would not be sufficient to maintain therapeutic levels for more than one or two days, if that long. Further injections would have to be given after a loading dose at intervals of four to 10 hours, depending upon the severity of the renal impairment. Also a large accumulation of penicillin cannot be expected to occur in the anuric patient given continuous therapy since the drug is still inactivated at a fairly rapid rate. Aqueous penicillin $\mathrm{G}$ may be expected to achieve the same prolongation of levels in the blood of anuric subjects as those achieved with procaine penicillin in the normal individual, but at higher serum concentrations after the same dose.

\section{Streptomycin}

Previous investigations have pointed out that in the presence of uremia streptomycin will accumulate in the blood if given continuously (21, 22). The data presented above indicate that a single dose given to a severely uremic patient may persist at reasonably high levels for many days. Several investigators (23-25) employing various methods of assay have reported that streptomycin is cleared primarily by glomerular filtration at rates varying from 30 to $70 \mathrm{ml}$. per minute. About 34 per cent of the drug is bound to plasma proteins (25) accounting for the fact that the clearance is less than that of the glomerular filtration rate. From 30 to 80 per cent of an intravenously administered dose has been recovered from the urine of normal individuals within the first 24 hours after injection (21-24, 26). Boxer and Jelinek (27) reported the serum half-life after intramuscular injection to be $2.72 \pm 0.47$ hours and similar values may be obtained by recalculation of the data reported by others $(24,26)$. The mean serum half-life of biologically active streptomycin determined in four normal men in the present study was two and four-tenths hours.

In view of these findings, one would expect the relation between streptomycin half-life in serum and the glomerular filtration rate to be somewhat similar although not identical to the findings reported for tetracycline (2). The half-life of streptomycin in anuric patients would appear from the present data to be similar to that of tetracycline. A conservative estimate of dosage increment necessary to maintain a reasonably high, but not excessive, serum level in a severely uremic patient can be suggested: following a loading dose of 1 to $2 \mathrm{Gm}$. of the drug, one-half the loading dose should probably be given every two to three days. The modification of dosage increments during the diuretic phase would not depend so much upon the amount of urine excreted as upon the recovery of renal function. For example, Patient $\mathrm{Su}$ had a very large urinary volume while maintaining fairly constant streptomycin levels 
after a single dose, and Boxer, Jelinek and Edison (25) have shown that the clearance of streptomycin is independent of the rate of urine flow.

\section{Erythromycin}

The half-life of erythromycin in the serum of the anuric patients was clearly longer than that observed in the normal subjects. However, the maximum value observed in a uremic patient was only five and eight-tenths hours. This indicates that although the kidneys are of some importance in the clearance of this drug from the body, nonrenal mechanisms may be of greater importance. A considerable amount of erythromycin is excreted into the bile (28-30) and ordinarily only about 15 per cent of an adminstered dose may be recovered in the urine of normal patients. Data on the clearance of erythromycin is limited, but would imply that a considerable amount of plasma binding is present (31). The rapid decay of erythromycin in the anuric patient indicates that the artificial kidney cannot be expected to have any therapeutic importance in clearing the drug from the blood. The anuric patient should, therefore, be treated with nearly the same dose and at about the same time intervals as a normal patient.

\section{Kanamycin}

Data on the pharmacology and therapeutic uses of kanamycin have been presented in an extensive report (32). Chemically, this antibiotic has much in common with streptomycin, and like the latter it is a fairly stable drug cleared primarily by glomerular filtration (33) and recoverable in large amounts in the urine $(34,35)$. The data in the two severely uremic patients presented in this paper indicate that this drug is retained in the serum in patients with renal disease for considerable periods of time. Since the drug has been shown to have nephrotoxic and ototoxic potential it would seem wise to avoid using this drug in patients with severe renal disease and certainly to avoid overdosage when it is used. If it is found necessary to give this drug to anuric patients, they should not receive increments more frequently than every three or four days and these increments should be approximately one-half of the loading dose used.

\section{Synthesis of data on antibiotics in renal disease}

With the exception of penicillin and the metabolic products of chloramphenicol (chiefly the monoglucuronide) the antibiotics currently in use are cleared by the kidney by glomerular filtration. The rate at which each antibiotic is cleared is dependent upon the extent to which it is bound to plasma proteins. Some of the antibiotics are metabolized or excreted by other channels at a rate so rapid that the contribution of the renal clearance may be almost negligible (chloramphenicol and erythromycin). The serum half-life of each antibiotic reflects the rate of both renal and nonrenal clearance mechanisms. Figure 3 depicts a hypothetical representation of the relation between renal function and the half-life of antibiotics in serum. Two hypothetical drugs are considered: Drug A, which is completely stable and cleared only by renal mechanisms, and Drug B, which is cleared entirely by nonrenal mechanisms. An arbitrary half-life in serum for individuals with normal renal function is established at five hours for each drug in this model.

The following considerations were taken into account in constructing Curve A: 1) The hypothetical substances, like inulin and mannitol, are not metabolized;2) the drug is removed entirely by renal mechanisms; 3) clearance is independent of the rate of urine flow; 4) serum half-life $(\mathrm{T} / 2)$ is determined after distribution and equilibration in body compartments has occurred; and 5) the relative volume distribution of the substance is independent of renal functional capacity. Then following the analysis presented by Newman, Bordley and Winternitz (36) in a study of the interrelation of the clearance of mannitol by the kidneys to the fall in plasma after a single intravenous injection we find that:

$$
\mathrm{C}=\mathrm{V}_{\mathrm{c}} \mathrm{S}
$$

where $C$ is the rate of renal clearance (here expressed as the per cent of normal renal function including all modes of excretion), $\mathrm{V}_{\mathrm{c}}$ is the relative volume distribution of the substance, and $S$ is the serum decay rate. Since:

$$
\mathrm{T} / 2=\frac{\log 2}{\mathrm{~S}},
$$




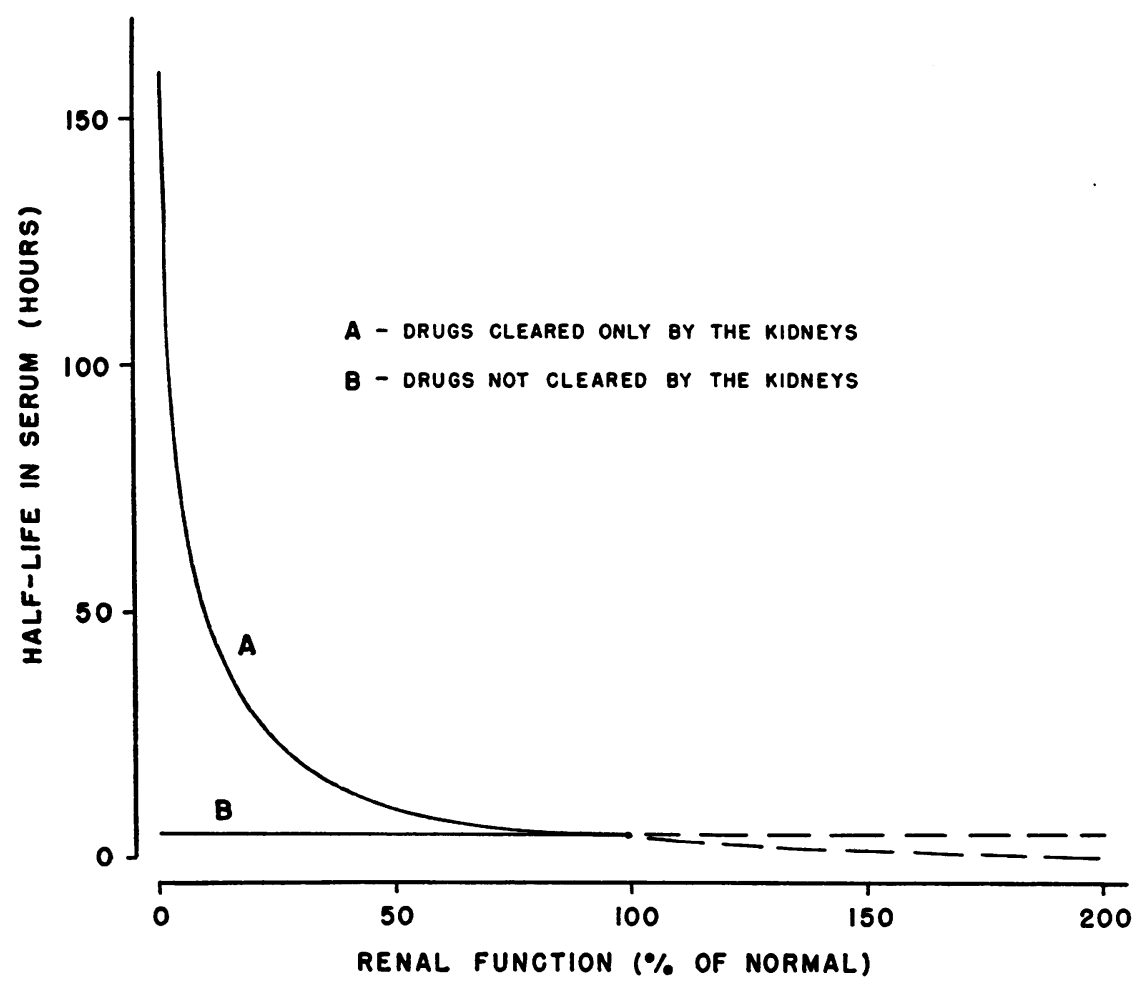

Fig. 3. Representation of Hypothetical Relationship Between Half-life of Drugs in Serum and Renal Function for Two Classes of Drugs

then

$$
\mathrm{S}=\frac{\log 2}{\mathrm{~T} / 2}
$$

and substituting in Equation 1 we find that

$$
\mathrm{C}=\frac{\mathrm{V}_{\mathrm{c}}(\log 2)}{\mathrm{T} / 2}
$$

The reciprocal relation between renal function and half-life in serum of the injected substance noted in Equation 4 permits construction of Curve $A$ in Figure 3. This shows that a marked prolongation of serum half-life does not occur until renal function has fallen below 20 to 30 per cent of normal, at which point the blood urea nitrogen and creatinine will also rise in the blood. Curve $\mathrm{B}$, on the other hand, reflects the fact that the serum half-life of Drug B is unaffected by the extent of renal dysfunction or hyperfunction.

Although drugs such as tetracycline, streptomycin, kanamycin and the chloramphenicol metabolites have characteristics in common with Drug A, they are excreted in part by nonrenal mechanisms as well. Although active chloramphenicol and to a lesser extent erythromycin and penicillin resemble Drug B in many respects, all of these drugs are excreted into the urine to varying extents. Thus, as was found in the studies of tetracycline (2) and chloramphenicol (3), each drug tends to resemble either Model A or B, but is identical with neither. The anuric patient permits an estimation of the half-life of the agent in serum which approximates that which may be expected in the absence of any renal excretory function, whereas the normal patient fixes the other extreme of the curve. By this approach the limitations of renal disease upon the persistence of a drug may be established.

These principles may also be useful in considering the effect of renal disease on the persistence of drugs other than antibiotics in the blood of patients with renal disease.

\section{SUM MARY}

The effect of severe renal disease on the persistence of penicillin, streptomycin, erythromycin and kanamycin in the blood has been studied by determining the rate of their decay in the serum. 
The serum half-life of penicillin was found to be prolonged from about one-half hour in normal subjects to seven to 10 hours in anuric patients, falling to four to seven hours during the early phase of recovery from acute renal failure. The role of the liver in the inactivation of penicillin was studied in two anuric patients who also had severe liver disease. The serum half-life in these patients was prolonged to 16.4 and 30.5 hours, respectively, suggesting that in the uremic patient, the liver may account for much of the degradation of the drug.

The half-life of streptomycin in the serum of anuric patients was found to be two to five days, falling to one to two days during diuresis. In one patient who became progressively uremic and subsequently died of acute renal tubular necrosis, streptomycin was still detectable 20 days after the last dose. ${ }^{8}$

The serum half-life of erythromycin was slightly prolonged from one and one-half hours in normal subjects to about five hours in three anuric patients. The presence of liver disease in one patient did not appear to prolong the serum halflife of erythromycin.

The serum half-life of kanamycin in two normal subjects was about four hours. Elevated levels of kanamycin were detectable in the blood of two severely uremic patients as long as four to five days following a single injection of $1.0 \mathrm{Gm}$.

A theoretical analysis of the effect of changing renal function on the serum half-life of antibiotics is presented; this may be applicable to other drugs as well.

\section{REFERENCES}

1. Kunin, C. M., and Rees, S. Persistence of antibiotics in blood of patients with acute tubular necrosis and uremia (abstract). J. clin. Invest. 1958, 37, 908.

2. Kunin, C. M., Rees, S. B., Merrill, J. P., and Finland, M. Persistence of antibiotics in the blood of patients with acute renal failure. I. Tetracycline

\footnotetext{
${ }^{8}$ Edwards and Whyte (37) recently reported observing five patients with renal failure who developed bilateral loss of vestibular function, with impairment in hearing in two of them, following a total of 4 to $14 \mathrm{Gm}$. of streptomycin. After tests in vitro showed that streptomycin could be removed by dialysis, they treated eight patients with acute renal failure by hemodialysis as early as possible after they had received various amounts of streptomycin. Only one of these eight developed signs of toxicity.
}

and chlortetracycline. J. clin. Invest. 1959, 38, 1487.

3. Kunin, C. M., Glazko, A. J., and Finland, M. Persistence of antibiotics in blood of patients with acute renal failure. II. Chloramphenicol and its metabolic products in the blood of patients with severe renal disease or hepatic cirrhosis. J. clin. Invest. 1959, 38, 1498.

4. Kunin, C. M., and Finland, M. Demethylchortetracycline. A new tetracycline antibiotic that yields greater and more sustained antibacterial activity. New Engl. J. Med. 1958, 259, 999.

5. Grove, D. C., and Randall, W. A. Assay Methods of Antibiotics. A Laboratory Manual. New York, Medical Encyclopedia, Inc., 1955.

6. Beyer, K. H. Pharmacological Basis of Penicillin Therapy. Springfield, Illinois, Charles C Thomas, 1950, Chapter II.

7. Eagle, H., and Newman, E. The renal clearance of penicillins $F, G, K$, and $X$ in rabbits and man. J. clin. Invest. 1947, 26, 903.

8. Humphrey, J. H. The excretion of penicillin in man. Nature (Lond.) 1944, 154, 765.

9. Barnett, H. L., McNamara, H., Shultz, S., and Tompsett, R. Renal clearances of sodium penicillin G, procaine penicillin $G$, and inulin in infants and children. Pediatrics 1949, 3, 418.

10. Huang, N. N., and High, R. H. Comparison of serum levels following the administration of oral and parenteral preparations of penicillin to infants and children of various age groups. J. Pediat. 1953, 42, 657.

11. Seeler, A. O., Wilcox, C., and Finland, M. Enhancement of blood levels by caronamide during intramuscular administration of penicillin. J. Lab. clin. Med. 1947, 32, 807.

12. Mathiesen, G. O., and Trolle-Lassen, C. Renal elimination of penicillin by the aged. Scand. J. clin. Lab. Invest. 1957, 9, 213.

13. Nichols, R. L., Jones, W. F., Jr., and Finland, M. Plasma penicillin levels from oral penicillins $V$ and $G$ and intramuscular penicillin G. Proc. Soc. exp. Biol. (N. Y.) 1955, 90, 688.

14. Unpublished data of Beyer, Boger and co-workers in Pharmacological Basis of Penicillin Therapy. Springfield, Illinois, Charles C Thomas, 1950, p. 152.

15. Rowlands, S., Rowley, D., and Stewart, H. C. Absorption and excretion studies with radioactive penicillin. Lancet 1948, 2, 493.

16. Eagle, $H$. The inactivation of penicillins $F, G, K$ and $\mathrm{X}$ by human and rabbit serum. J. exp. Med. 1947, 85, 141.

17. Benedict, R. G., Schmidt, W. H., and Coghill, R. D. The stability of penicillin in aqueous solution. $\mathrm{J}$. Bact. 1946, 51, 291.

18. Anderson, W. H., and Brodersen, R. The elimination of penicillin $G$ in bilaterally nephrectomized dogs. J. clin. Invest. 1949, 28, 821. 
19. Spitzy, K. H., and Hitzenberger, G. The distribution volume of some antibiotics. Antibiot. Ann 1957-1958, 996.

20. Bull, G. M. Uraemias (Goulstonian Lecture). Lancet 1955, 1, 777.

21. Rake, G., and Donovick, R. Absorption, distribution and excretion of streptomycin in Streptomycin: Its Nature and Practical Application, S. A. Waksman, Ed. Baltimore, Williams and Wilkins Co., 1949.

22. Buggs, C. W., Pilling, M. A., Bronstein, B., and Hirshfeld, J. W. The absorption, distribution and excretion of streptomycin in man. J. clin. Invest. 1946, 25, 94.

23. Marshall, E. K., Jr. The absorption, distribution and excretion of streptomycin. J. Pharmacol. exp. Ther. 1948, 92, 43.

24. Adcock, J. D., and Hettig, R. A. Absorption, distribution and excretion of streptomycin. Arch. intern. Med. 1946, 77, 179.

25. Boxer, G. E., Jelinek, V. C., and Edison, A. O. Streptomycin: Clearance and binding to protein. J. Pharmacol. exp. Ther. 1949, 97, 93.

26. Anderson, D. G., and Jewell, M. The absorption, excretion and toxicity of streptomycin in man. New Engl. J. Med. 1945, 233, 485.

27. Boxer, G. E., and Jelinek, V. C. Streptomycin in the blood: Chemical determinations after single and repeated intramuscular injections. J. Pharmacol. exp. Ther. 1948, 92, 226.

28. Griffith, R. S., Johnstone, D. M., and Smith, J. W. The distribution and excretion of erythromycin following intravenous injection. Antibiot. Ann. 1953-1954, 496.
29. Takimura, Y., and Lopez-Belio, M. Excretion of erythromycin through the biliary tract. Antibiot. Med. 1955, 1, 561.

30. Twiss, J. R., Berger, W. V., Gillette, L., Aronson, A. R., and Siegel, L. The biliary excretion of erythromycin (Ilotycin). Surg. Gynec. Obstet. 1956, 102, 355.

31. Lee, C-C., Anderson, R. C., and Chen, K. K. Renal clearance of erythromycin. Proc. Soc. exp. Biol. (N. Y.) 1955, 88, 584.

32. Finland, M. (Conference chmn.), et al. The basic and clinical research of the new antibiotic, kanamycin. Ann. N. Y. Acad. Sci. 1958, 76, 17.

33. Tisch, D. E., Huftalen, J. B., and Dickison, H. L. Pharmacological studies with kanamycin. Ann. N. Y. Acad. Sci. 1958, 76, 44.

34. Welch, H., Wright, W. W., Weinstein, H. I., and Staffa, A. W. In vitro and pharmacological studies with kanamycin. Ann. N. Y. Acad. Sci. 1958, 76, 66.

35. White, A., and Knight, V. Kanamycin: Pharmacological, microbiological and clinical observations. Ann. N. Y. Acad. Sci. 1958, 76, 277.

36. Newman, E. V., Bordley, J., III, and Winternitz, J. The interrelationships of glomerular filtration rate (mannitol clearance), extracellular fluid volume, surface area of the body, and plasma concentration of mannitol. A definition of extracellular fluid clearance determined by following plasma concentration after a single injection of mannitol. Bull. Johns Hopk. Hosp. 1944, 75, 253.

37. Edwards, K. D. G., and Whyte, H. M. Streptomycin poisoning in renal failure: An indication for treatment with an atrificial kidney. Brit. med. J. 1959, $1,752$. 